\title{
The relationship between publication volume of biomedical research and burden of disease
}

\section{Authors}

Niels Hagenaars, $\mathrm{PhD}^{1 *}$, Thijs de Kruif MSc ${ }^{1}$, Lissy van de $\operatorname{Laar}^{1}$, MSc, Ludo Waltman ${ }^{2}, \mathrm{PhD}$, Ingeborg Meijer, $\mathrm{PhD}^{2}$, Marcel Levi, $\mathrm{MD} \mathrm{PhD}^{3,4}$, Anshu Gupta, $\mathrm{PhD}^{1}$

*Corresponding author

Niels Hagenaars

Gupta strategists

PO Box 16

4060 GA Ophemert

Netherlands

niels.hagenaars@gupta-strategists.nl

Ingeborg Meijer

Centre for Science and Technology Studies (CWTS), Leiden University

Kolffpad 1

2333 BN Leiden

Netherlands

i.meijer@cwts.leidenuniv.nl

1) Gupta Strategists, PO Box 16, 4060 GA Ophemert, The Netherlands

2) Centre for Science and Technology Studies, Leiden University, The Netherlands

3) University College London Hospitals NHS Foundation Trust, London, United Kingdom.

4) Academic Medical Centre, University of Amsterdam, The Netherlands. 


\section{ABSTRACT \\ Background}

One could hypothesize that the biomedical research agenda aligns with the (global) burden of disease, since it should aim to reduce illness and death. We investigated the relationship between publication volume and Research-dependent Fraction of Global Burden of Disease (BoD-RdF): the fraction of Burden of Disease that can only be relieved by further biomedical research. Aim of this comparison is to evaluate and support the optimization of biomedical research portfolios.

\section{Methods}

BoD-RdF, defined as the burden of disease reported in the $1^{\text {st }}$ quartile of the age corrected burden of disease per capita for all countries, was calculated using 2014 Global Burden of Disease data from WHO for the years 2000 and 2012. Publication volumes were based on 6.5 million publications between 2000 and 2012 from the Clarivate Analytics’ Web of Science database, categorized into disease groups by keyword analysis and text mining techniques. The categorized publication volumes were compared to BoD-RdF.

\section{Findings}

From the global burden of disease in 2012, we estimate that $48 \%$ depends on further biomedical research to be relieved (BoD-RdF). Generally, diseases with high burden are underresearched, whereas diseases with relatively little burden are overrepresented in the global research portfolio. Large variations in publication volume (up to twelve-fold) exist for diseases with similar BoD-RdF. Likewise, diseases with a similar publication volume vary greatly in BoDRdF (up to 50-fold). Compared to 2000, the disease-specific publication volume almost doubled in 2012, but the correlation with burden of disease and BoD-RdF showed only a small improvement.

\section{Interpretation}

We found a profound and persistent misalignment of publication volume with BoD-RdF. For 18 diseases, which account for $50 \%$ of the global BoD-RdF, a factor 2 increase of the publication volume would be justified based on BoD-RdF. This misalignment persisted over the last decade, despite doubling of the total number of disease specific publications.

Although we recognize that the global research portfolio will never be dictated by global disease burden alone, we propose that the comparison between BoD-RdF and publication volume provides supportive insights that can be used in optimization of the research portfolio.

\section{Funding}

This research received no specific grant from any funding agency in the public, commercial or not-for-profit sectors. The authors are solely responsible for study design, data collection and analysis, interpretation, and writing of the report. 


\section{BACKGROUND}

Biomedical research is the broad area of science that aims to find ways to prevent, effectively diagnose and treat diseases that cause illness and death in humans. Hence, it might be hypothesized that the biomedical research agenda would be based on the (global) burden of disease. In other words, diseases with a relatively high burden in terms of morbidity and mortality should ideally get proportionately more research. Studying the alignment of burden of disease with biomedical research output may contribute to an objective evaluation of the current biomedical research portfolio.

We were surprised that, despite the enormous amount of biomedical research and funding, and the vast amount of literature on quality of biomedical research on the one hand, and the comprehensive WHO study on global burden of disease on the other hand ${ }^{1}$, little research has been published on the relationship between the global disease-specific research portfolio and the burden of disease ${ }^{2,3}$.

Classification of publications to broader disciplines (e.g. neurology, cardiology, surgery) is readily available. However, a more incisive analysis can be achieved by analyzing systematic classification of diseases according to ICD-10 codes employing MESH terminology or text mining techniques and author keywords, as we have recently developed ${ }^{4}$. Using the latter technique, mapping research portfolios (based on research output in publications and citations by disease) can be done systematically, on a global, national, and institutional level over time.

The burden of disease for a specific disease can be alleviated in two ways. Firstly, current international best practices for prevention, diagnosis, and treatment of a disease can be implemented worldwide. Secondly, new scientific insights can improve the current best practice disease management strategies and further alleviate burden of disease. For diseases with a large variation in burden of disease across countries, such as HIV/AIDS, we hypothesize that the global burden of disease could largely - but not entirely - be reduced by implementing best practices rather than developing new scientific insights. For diseases with little variation in burden of disease between countries, such as depression, we hypothesize that, for improving the global burden of disease, new medical breakthroughs are required. When studying the alignment of the publication volume and burden of disease between diseases, we need to take both these factors into account. Or more specifically, disease burden that could be alleviated by implementing existing disease management strategies rather than by new research should be excluded when studying alignment with research priorities.

Currently a limited list of amenable diseases, originally developed by Rutstein and colleagues ${ }^{5}$, is widely used as a measure for avoidable burden of disease ${ }^{6}$. Our goal was to study the burden of all diseases in all countries and relate this to disease-specific biomedical research. Therefore, we introduce a new measure that we propose to use as a proxy for the burden that can only be relieved by further biomedical research: Research-dependent Fraction of Global Burden of Disease (BoD-RdF). We believe that our analysis of the relationship between BoD-RdF and the volume of disease-specific research ${ }^{7}$ can contribute to better alignment of biomedical research portfolios. 


\section{METHOD}

\section{Burden of Disease}

We used data from the 2014 Global Burden of Disease study for all diseases in all countries ${ }^{1}$. The same source was used for the number of inhabitants and age distribution per country. We expressed burden of disease as DALYs per 1000 capita to correct total burden for differences in population size. We used data on burden of disease and publication volume per disease for both 2000 and 2012.

\section{Research-dependent Fraction of Global Burden of Disease}

The BoD-RdF represents the fraction of a disease's total burden of disease that can only be relieved by further biomedical research. For diseases with a high variation in burden per capita between countries, the BoD-RdF is low. Alleviation of most of the burden requires implementation of best practices rather than new biomedical research. For diseases with little variation across countries, the BoD-RdF is high and further biomedical research plays an important role in alleviating the global burden. Burden of disease per capita per country should be age corrected for fair comparison. The variation in Burden of disease per capita between countries for age specific diseases such as dementia is highly dependent on a country's age distribution. Therefore, we defined BoD-RdF as the burden of disease reported of the $1^{\text {st }}$ quartile of the age corrected burden of disease by country.

For example, the age corrected burden of disease for diabetes mellitus, ranges between countries from 2.4 DALY's per 1000 capita in the UK to 48.5 in Mauritius. The burden reported in the $1^{\text {st }}$ quartile (6.3 DALY's per 1000 capita) is the BoD-RdF for diabetes mellitus. The $1^{\text {st }}$ quartile was chosen because it reflects an international best practice exhibited by several countries, whereas the lowest possible burden might reflect a statistical outlier. While this definition is arbitrary and other, more sophisticated, approaches may be better; it intuitively distinguishes between what can be achieved with current knowledge and where further knowledge is needed.

\section{Disease specific publication volume}

Publication volumes for biomedical research were based on Clarivate Analytics' Web of Science database ${ }^{7}$ (WoS) as available at the Centre for Science and Technology Studies (CWTS) of Leiden University. We included biomedical research fields only and compiled a dataset with 6.5 million publications, published between 2000 and early 2014. Using text mining techniques and analysis of keywords, publications were linked to 269 disease groups. This method and its validation is described in detail elsewhere ${ }^{4}$.

Correlations between burden and publication volume were calculated using the $\mathrm{R}^{2}$-method based on a simple linear regression analysis with BoD-RdF as the dependent variable.

\section{FINDINGS}




\section{Research dependent fraction of global burden is $\mathbf{4 8 \%}$ of global burden of disease, but varies greatly between diseases}

Table 1 provides the number of publications, global burden of disease and BoD-RdF per disease for both 2000 and 2012.

\section{[TABLE 1]}

$48 \%$ of the global burden of disease is BoD-RdF. The share of BoD-RdF in the global burden of disease varies widely between diseases. As illustrated in Figure 1 for the 25 diseases that cause the most burden worldwide, the BoD-RdF varies between 2.2\% for HIV-AIDS and $99.6 \%$ for depression.

\section{[FIGURE 1]}

\section{Biomedical research volume is correlated to BoD-RdF with large variation between diseases}

Generally, diseases with higher BoD-RdF have a higher volume of global research output $\left(\mathrm{R}^{2}=0.431\right.$, Figure $\left.2 \mathrm{~A}\right)$. There is a large variation between diseases. Compared to a theoretical direct proportionality between BoD-RdF and publication volume, many diseases with high BoD-RdF are relatively underresearched (indicated in green in Table 1 and Figure 2A), whereas other diseases with relatively low BoD-RdF are overrepresented in the global diseasespecific research portfolio (indicated in blue in Table 1 and Figure 2A), giving a weak linear correlation (see Figure 2A and data in Table 1).

\section{[FIGURE 2]}

Comparing publication volume to global burden of disease instead of BoD-RdF gives a similar correlation $\left(\mathrm{R}^{2}=0.433\right.$, data in Table 1$)$.

As shown in Table 1, there are big differences between the share in research output and the share in burden of disease for specific diseases. The data from Table 1 is illustrated in Figure 2B to highlight these large differences. For example, diabetes and back and neck pain have a similar BoD-RdF (2.15\% and $1.96 \%$ of the total global BoD-RdF, respectively), but the publication volume on diabetes is twelvefold higher than back and neck pain (3.77\% versus $0.30 \%$, respectively). Similarly, 'melanoma and other skin cancers' has a comparable publication volume to lower respiratory infections $(1.01 \%$ and $0.86 \%$ of the global publication volume, respectively) but causes a much lower global burden ( $0.10 \%$ versus $5.36 \%)$.

To understand if this mismatch reflects different cultures between biomedical disciplines, like publishing a single comprehensive paper or multiple papers on a single scientific finding, we delve deeper into a single discipline. We find that this mismatch is also present within biomedical disciplines, such as in the well-researched discipline of oncology. Within oncology certain cancers are overresearched and others underresearched in relation to their global BoD- 
RdF. For example, breast cancer, lymphoid cancers, colorectal cancer, liver cancer and stomach cancer cause a similar BoD-RdF (between 2.47 and 2.98 DALYs per 1000 capita worldwide in 2012), as illustrated in Figure 2C. The publications volumes for these diseases however differ 7-fold. Similarly, the publication volume for melanoma, prostate cancer, colon cancer and lung cancer is similar (between approximately 5000 and 6200 publications in 2012), but their burden varies 15 -fold.

\section{Research portfolio is slightly better aligned to BoD-RdF in $\mathbf{2 0 1 2}$ than in $\mathbf{2 0 0 0}$}

The 2012 BoD-RdF and publication volume show a similar pattern to that in 2000 (see Table 1). The correlation of Bod-RdF and publication volume was slightly better in $2012\left(\mathrm{R}^{2}=0.431\right)$ than in $2000\left(R^{2}=0.367\right)$. The disease-specific research volume doubled between 2000 and 2012 from approximately 275000 to 550000 publications, while the BoD-RdF has marginally declined from 200 to 185 DALYs per 1000 capita in the same period. The diseases that account for a high publication volume in 2012 are largely the same as in 2000.

In general, the share in publication volume changed in line with the share of BoD-RdF for most diseases. However, for some diseases an opposite trend was observed. For example, neonatal conditions and tuberculosis decreased in share of BoD-RdF, -1.44 and -0.20 percentage point respectively, while their share in publication volume increased, +0.22 and +0.09 percentage point. Conversely, HIV/AIDS and hypertensive heart disease increased in share of BoD-RdF, +0.03 and +0.09 percentage point respectively, while their share in publication volume decreased, -0.33 and -0.15 percentage point.

\section{INTERPRETATION}

Our results show that BoD-RdF is not a strong driver for publication volume catalogued by disease. There is a weak correlation, and the misalignment is profound. For 18 diseases, a doubling in the current research volume is justified based on their burden (see green triangles in Figure 2A). These 18 diseases together account for more than $50 \%$ of the total global burden of disease. For Migraine and headache, hyperplasia of the prostate, osteoarthritis, back and neck pain, and other congenital anomalies (marked bold in Table 1), a fourfold increase in publications is required to bring the research volume in line with BoD-RdF. For 28 diseases the 2012 publication volume could be halved if we base our research portfolio on BoD-RdF alone (see red dots in Figure 2A). These 28 diseases account for 18\% of the total global burden of disease.

The misalignment is persistent over the last decade and is therefore likely to persist in the near future if not actively corrected. We conclude that despite almost doubling of the research volume in the period 2000-2012, the pattern of overresearched and underresearched diseases has remained essentially the same. Our analysis provides policy makers, funding organizations, as well as researchers a quantitative source of information for realigning biomedical research priorities with the goal to alleviate burden of disease. 
If priorities in research were based on BoD-RdF alone, one would have greater impact in reducing global burden. Here we assume that volume of publication ultimately drives reduction in burden of disease. We have no direct proof of this, but it seems reasonable to expect that more research would eventually lower research dependent burden. We also assume that we can work with publication volume rather than research effort and funding. We have not shown that additional funding or effort results in more publications, though again this seems reasonable. Other studies have explored the match between funding and burden of disease, albeit with a narrower focus on NIH funding alone ${ }^{5}$.

To derive a better research portfolio, we draw a theoretical linear reference line where the publication volume is directly proportional to BoD-RdF (a forced fit through $(0,0)$ and $(1,1)$ coordinates: see blue line in Figure 2A). Deviation from the line represents the extent to which a disease area is over- or underresearched compared to its BoD-RdF. In a 'zero sum game' exercise, where the total publication volume remains constant, we could shift the research focus and thus publication volume from overresearched diseases where publication volume is relatively high in relation to BoD-RdF, to underresearched diseases where the opposite is the case. Of the approximate total of 550000 publications we attributed to diseases in 2012, we find that $\sim 400000$ publications focus on overresearched diseases and $\sim 150000$ cover underresearched diseases. In Table 1, the major diseases which are overresearched are highlighted in blue, whereas the green colored diseases merit more research.

A publication volume portfolio driven solely by BoD-RdF would be certainly different from what we had in 2012. Diseases like back and neck pain, migraine and headache, and osteoarthritis would deserve 5 to 12 times more publications than the 2012 volume, while infertility and multiple sclerosis could do with a much lower volume -half the 2012 publication volume. This mismatch can be found within every discipline.

Of course, there are limitations to our study:

1. Disease burden in certain disease areas can partly be eradicated by applying existing biomedical knowledge. For this part of the burden, more research is not needed but rather implementation of best practices is required. We have tried to correct for this effect in our methodology, by using BoD-RdF instead of burden of disease, but this is an approximation.

2. Classifying $\sim 6.5$ millions publications and linking these to 269 disease areas will inevitably contain inaccuracies, even though we validated the linking methodology thoroughly ${ }^{4}$, we cannot be sure how this may influence these results.

3. Not all fundamental research publications that may have contributed to alleviating burden of disease can be attributed to specific diseases. This could introduce a bias in the attribution of publications to diseases.

Taking these limitations into consideration, there are several other factors that may explain the observed misalignment between biomedical publication volume and burden of disease. 
1. The research portfolio is driven by many other factors than burden of disease, like political and funding priorities, commercial opportunities, geographical or individual preferences, and leading trends in research ${ }^{8,9,10}$.

2. The impact of research on reducing burden of disease may vary between diseases. Diseases likely have different relations of research effort vs. burden of disease reduction. Some diseases may therefore require more research efforts to achieve the same reduction in burden. Studying longer timelines and trying to account for multiple factors in a multivariate analysis could provide insights in these differences, potentially uncovering in which disease areas biomedical publications contribute less to better health. This could be a useful, albeit challenging, avenue of future research.

3. The time lag between research initiation and publication may be significantly different from the burden of disease progression for the same disease, resulting in the observed mismatch. Burden of a disease may increase or decrease unpredictably, for example due to epidemics, changes in lifestyle, age distribution and medical breakthroughs, resulting in research misalignment. This effect could be assessed by epidemiological studies and can be incorporated in designing research priorities.

4. The geographical mismatch between research (mainly developed countries) and burden (mainly developing countries) could also partially explain the observed mismatch between global BoD-RdF and global publication volume. We undertook country specific analyses (not presented here) and found that similar levels of mismatch exist at a country level as we report here at a global level, though not for all countries.

While recognizing the limitations and potential explanations, we believe that the comparison between BoD-RdF and publication volume is a relevant metric that can already be used to support better alignment of the biomedical research portfolio. Although we cannot quantify how much extra reduction in DALY is possible if we realign the research portfolio, we can be confident that for certain disease areas more research is justified and can be undertaken by shifting the priorities between diseases.

\section{FUNDING}

This research received no specific grant from any funding agency in the public, commercial or not-for-profit sectors. The authors are solely responsible for study design, data collection and analysis, interpretation, and writing of the report.

\section{Individual contributions}

$\mathrm{NH}$ and AG wrote the manuscript together with LL, TK and ML. TK performed the analyses. $\mathrm{NH}, \mathrm{AG}, \mathrm{LL}$ and TK performed the literature review. LL and NH worked on the definitions of disease groups, categorized the author keywords, and made the disease specific keywords. IM gave access to the WoS database for collaborative research and reviewed the manuscript. LW performed the classification of publications to disease groups based on a text mining algorithm and keyword analysis and reviewed the manuscript.

\section{Declaration of interests}


We have read and understood the Lancet policy on declaration of interests and declare no competing interests. 


\section{Evidence before this study}

Studies on both burden of disease and research portfolios by disease classification are of recent origin. Most of the work emerged at the end of last century. The Global Burden of Diseases, Injuries and Risk Factors study today is a comprehensive study of 195 countries and territories from 1995-2015. The value and limitations of the GBD-study are well documented elsewhere 11-14. More recent work builds on the opportunities such a comprehensive database provides.

A recent study by Murray and colleagues ${ }^{15}$ combines the GBD data with amenable mortality studies to develop a composite healthcare access and quality index for all countries. This study presents frontiers for 32 disease areas and for all countries. Amenable mortality is a useful concept with a longer history of study dating from Rutstein and colleagues in the $1970 \mathrm{~s}^{5}$ to a recent review by Nolte and $\mathrm{McKee}^{6}$. By its very nature such an approach is limited. We are not aware of any comprehensive study that includes all diseases, and even if there is agreement on a definition of amenability of mortality, it is a constantly changing target. Nolte and McKee acknowledge these limitations in their review and caution against use of this definition for comparative purposes. Through its frontier analysis, the work of Murray and colleagues provides a more robust and comprehensive approach to amenability but it is limited to the 32 causes amenable to treatment.

Literature is more limited on classification, quantification and qualification of biomedical research output, or deciphering research portfolios. We are aware of three studies. Two of these use NIH funding in the USA per disease area. Gross and colleagues ${ }^{8}$ were the first to publish the link between NIH funding (1996) and burden of disease (1994) and Gillum and colleagues updated their work to 2004-2006 ${ }^{9}$. Both found that burden of disease is a predictor of funding though there were large outliers. These studies are limited to the USA and to a single albeit major funding source, the NIH. Furthermore, the authors acknowledge that the NIH funding mapping to diseases is not standardized and its reliability is unknown. Nonetheless the authors found that in the ten-year period between the two studies the alignment between funding and burden of disease had not improved.

A study by Evans and colleagues reports a methodology to link publications to diseases and to compare publication volume to burden of disease ${ }^{2}$. This study comes closest to our own work here, though the focus of the study is on the mismatch between the burden and health knowledge in developing and developed countries. Although Evans et al. use burden of disease, they do not split it in an 'amenable' and 'unamenable' component.

\section{Added value of this research}

The research we present is novel in several ways. Other than Evans and colleagues this is the first reported study that links all Web of Science biomedical publications (2.5 million) to a comprehensive and detailed disease classification based on ICD-10 (269). We have used two base years 2000 and 2012, a much longer and recent period than reported so far. 
Importantly, we have used the observed variation in burden of diseases across nations in the GBD study to split the burden in a medically 'amenable' and an 'unamenable' component (BoD-RdF). This allows us to correct for areas where the publication volume appears to be too low given the burden while most likely more biomedical research will not have much effect on the burden.

To our knowledge this study is unique both in comprehensiveness and systematic approach and in its use of the country variation to identify the BoD-RdF. 


\section{REFERENCE LIST}

1 WHO. Global Burden of Disease Study 2014. 2014.

2 Evans JA, Shim JM, Ioannidis JPA. Attention to local health burden and the global disparity of health research. PLoS One 2014; 9. DOI:10.1371/journal.pone.0090147.

3 Moses H, Matheson DHM, Cairns-Smith S, George BP, Palisch C, Dorsey ER. The Anatomy of Medical Research. JAMA 2015; 313: 174.

4 van de Laar L, de Kruif T, Waltman L, Meijer I, Gupta A, Hagenaars N. Improving the evaluation of worldwide biomedical research output: classification method and standardized bibliometric indicators by disease. BMJ Open., accepted for publication

5 Rutstein DD, Berenberg W, Chalmers TC, et al. Measuring the Quality of Medical Care. N Engl J Med 1976; 294: 582-8.

$6 \quad$ Nolte E, McKee M. Does Health Care Save Lives? Avoidable Mortality Revisited. 2004 DOI:ISBN 1-902089-94-4.

7 Reuters T. No Title. Web Sci. 2015. www.webofknowledge.com, (accessed April 6, 2015).

8 Gross CP, Anderson GF, Powe NR. The Relation between Funding by the National Institutes of Health and the Burden of Disease. $N$ Engl J Med 1999; 340: 1881-7.

9 Gillum LA, Gouveia C, Dorsey ER, et al. NIH disease funding levels and burden of disease. PLoS One 2011; 6. DOI:10.1371/journal.pone.0016837.

10 Kinge JM, Roxrud I, Vollset SE, Skirbekk V, Røttingen J-A. Are the Norwegian health research investments in line with the disease burden? Health Res Policy Syst 2014; 12: 64.

11 Wang H, Naghavi M, Allen C, et al. Global, regional, and national life expectancy, allcause mortality, and cause-specific mortality for 249 causes of death, 1980-2015: a systematic analysis for the Global Burden of Disease Study 2015. Lancet 2016; 388: 1459-544.

12 Forouzanfar MH, Afshin A, Alexander LT, et al. Global, regional, and national comparative risk assessment of 79 behavioural, environmental and occupational, and metabolic risks or clusters of risks, 1990-2015: a systematic analysis for the Global Burden of Disease Study 2015. Lancet 2016; 388: 1659-724.

13 Vos T, Allen C, Arora M, et al. Global, regional, and national incidence, prevalence, and years lived with disability for 310 diseases and injuries, 1990-2015: a systematic analysis for the Global Burden of Disease Study 2015. Lancet 2016; 388: 1545-602.

14 Kassebaum NJ, Arora M, Barber RM, et al. Global, regional, and national disabilityadjusted life-years (DALYs) for 315 diseases and injuries and healthy life expectancy (HALE), 1990-2015: a systematic analysis for the Global Burden of Disease Study 2015. Lancet 2016; 388: 1603-58.

15 Murray C. Healthcare Access and Quality Index based on mortality from causes amenable to personal health care in 195 countries and territories, 1990-2015: a novel analysis from the Global Burden of Disease Study 2015. Lancet 2017; 17: 30818-8. 


\section{List of figures}

\section{TABLES AND FIGURES}

Table 1: Global publications, burden of disease, and BoD-RdF for a set of 77 diseases/causes in 2000 and 2012, and the share of BoD-RdF of the total burden of disease and share of BoDRdF divided by the share of publications in 2012.

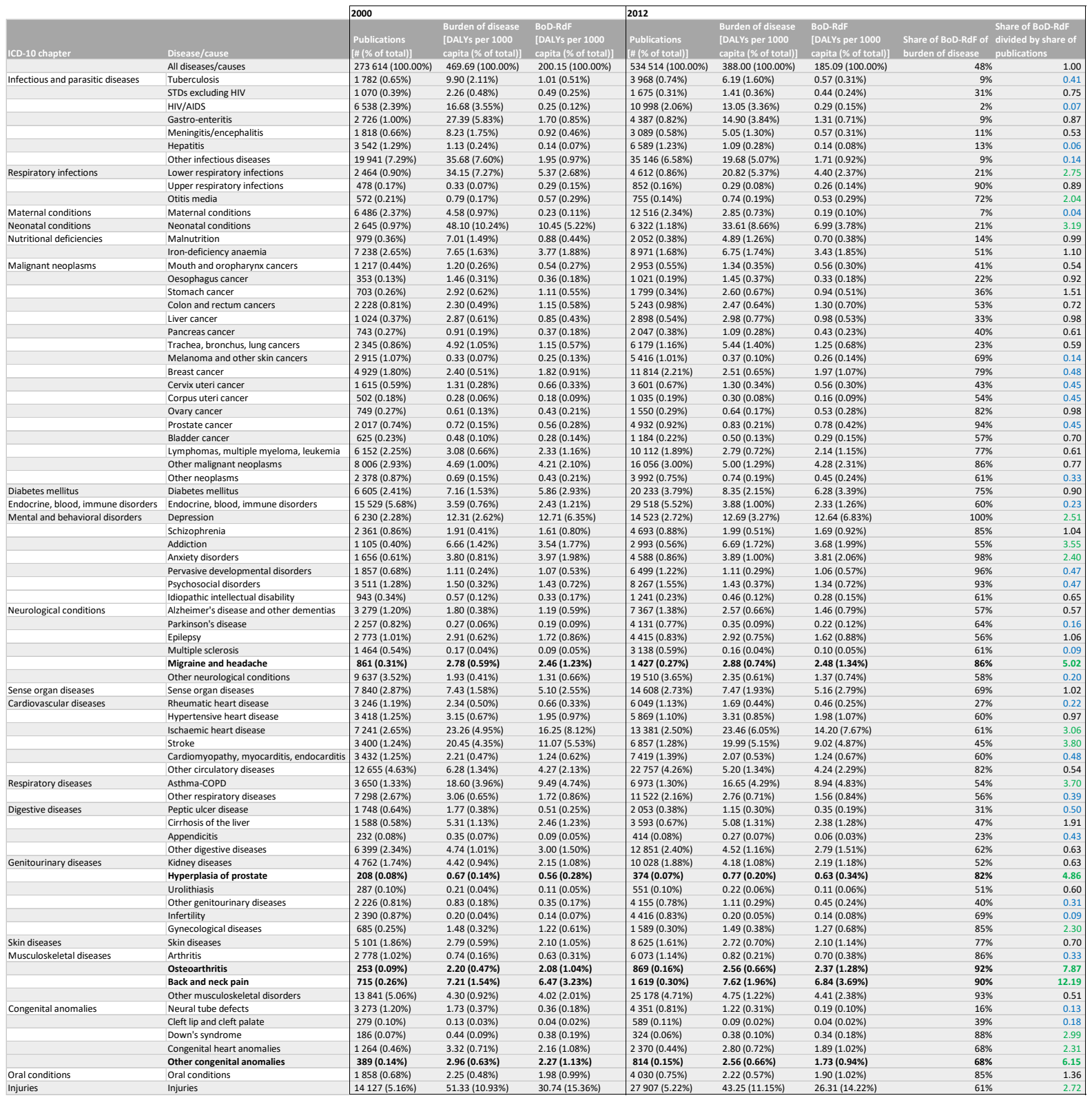


A

$\square$ Research dependent Fraction of Burden of Disease (BoD-RdF)

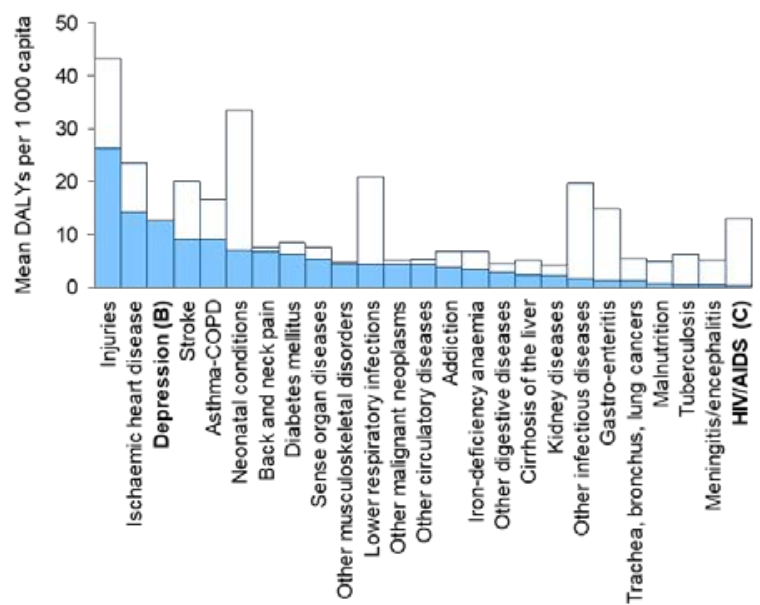

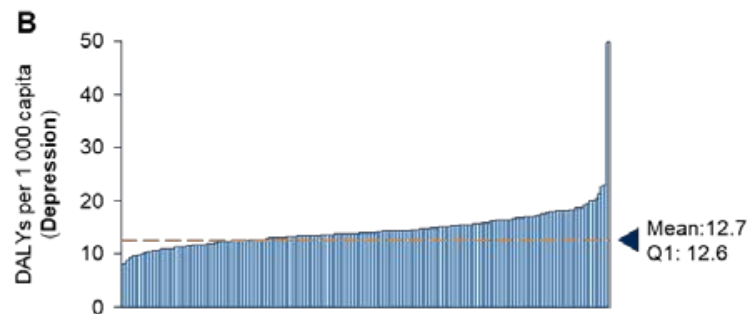

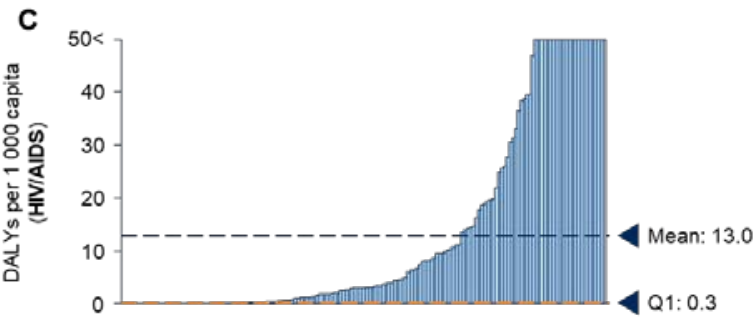

Figure 1: Burden of disease and the research dependent fraction of burden of disease (BoD$\mathrm{RdF})$. The figures show the age-corrected burden per country: regional burden of disease by age-group projected on the global age-distribution. (A) Burden of the 25 diseases causing most burden of disease globally (B) Burden of depression by country (C) Burden of HIV/AIDS by country.
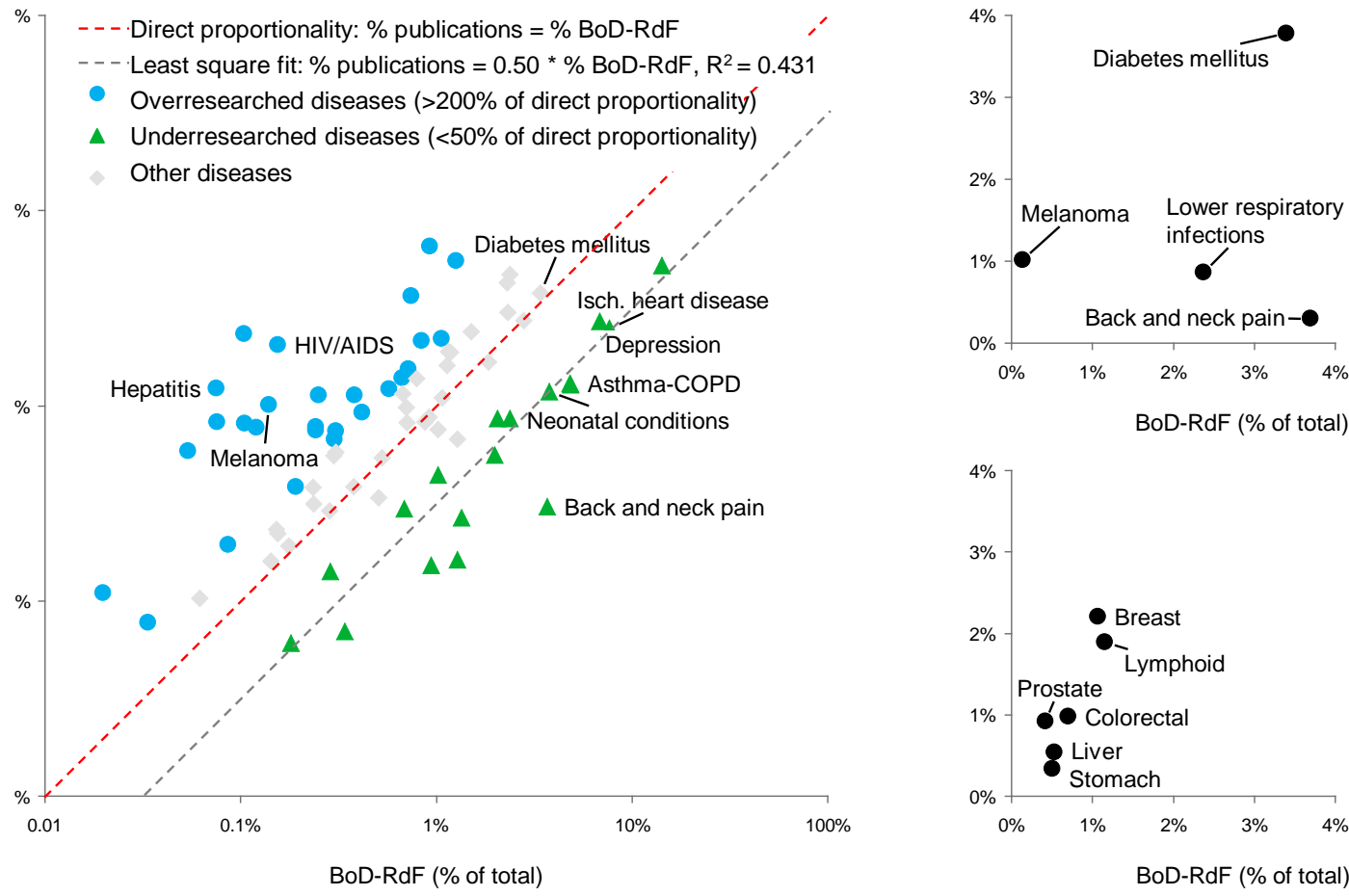

Figure 2: Share of BoD-RdF versus share of biomedical publications by disease in 2012. (A) All 77 causes/diseases (B) Illustrative selection of specific diseases with comparable publication volume but very different BoD-RdF (melanoma and lower respiratory infections) and diseases with comparable BoD-RdF but very different publication volumes (diabetes 
mellitus and back and neck pain) (C) Illustrative selection of specific cancers with comparable BoD-RdF but a large variation in publication volume. 